\title{
Sonourethrogram in comparison with retrograde urethrogram in evaluation of male anterior urethra
}

\author{
Haneef D. ${ }^{1 *}$ \\ DOI: https://doi.org/10.17511/ijmrr.2019.i05.07
}

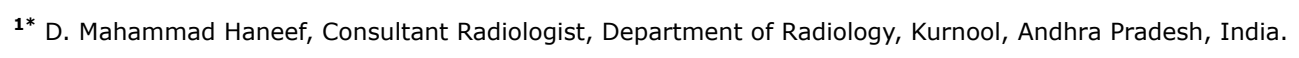

\begin{abstract}
Objective: Imaging of anterior male urethra is usually done using retrograde urethrogram, which utilises radiation. This study utilises high resolution ultrasound which is radiation free and helps to understand urethral pathologies like strictures, chronic urethritis etc. This study also compares the utility of sonourethrogram with retrograde urethrogram and provides the advantages of using sonourethrogram. Also establishes the drawbacks of using sonourethrogram over retrograde urethrogram. Material \& Methods: This study was done on 60 patients, came with different complaints like difficulty in micturition, obstructed urinary stream, pain in penis, increasing frequency of micturition and referred to us for retrograde urethrogram. Patients were examined with high resolution ultrasound with due permissions. Results are compared with findings of retrograde urerthrogram. Results: Out of 60 patients, 59 had strictures shown in retrograde urethrogram either in penile, bulbar or membranous parts. Only one patient had membranous urethral stricture in retrograde urethrogram which was not shown in sonourethrogram. All other patients have shown strictures in sonourethrogram. One patient had penile urethral calculus shown in both sonourethrogram and retrograde urethrogram. Conclusion: Sonourethrogram is equally effective as that of retrograde urethrogram especially in anterior urethral strictures. However, it underestimates or less useful in evaluation of posterior male urethral pathologies. Sonourethrogram is easily available, cost effective technique that uses no radiation in the evaluation of male urethra. It is equally effective compared to retrograde urethrogram in the evaluation of male anterior urethral strictures. Periurethral pathologies like fibrosis are better evaluated with sonourethrogram than with retrograde urethrogram. It is also important in the surgical planning and approach.
\end{abstract}

Keywords: Retrograde Urethrogram, Stricture, Sonourethrogram, Urethra

Corresponding Author

D. Mahammad Haneef, Consultant Radiologist, Department of Radiology, Kurnool, Andhra Pradesh, India.

Email: haneeef88@gmail.com
How to Cite this Article

To Browse

Haneef DM. Sonourethrogram in comparison with retrograde urethrogram in evaluation of male anterior urethra. Int J Med Res Rev. 2019;7(5):389395.

Available From

https://ijmrr.medresearch.in/index.php/ijmrr/article/ view/1084

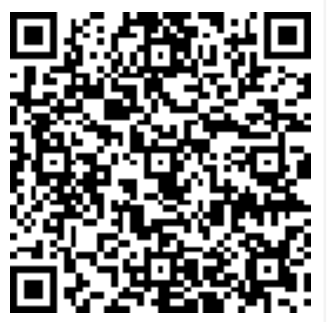

Manuscript Received 2019-09-10

Conflict of Interest No
Review Round 1 2019-09-20

Funding

$\mathrm{Nil}$

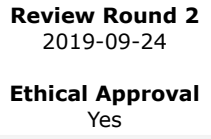

Ethical Approval Yes

Review Round 3

Plagiarism X-checker $8 \%$
Accepted 2019-09-27

Note

(c) 2019 by D. Mahammad Haneef and Published by Siddharth Health Research and Social Welfare Society. This is an Open Access article licensed under a Creative Commons Attribution 4.0 International License https://creativecommons.org/licenses/by/4.0/ unported [CC BY 4.0]. 


\section{Introduction}

Usually pathologies pertaining to urethra cause difficulty in micturition, obstructed urinary stream, pain while passing urine. They are usually result of chronic infection, trauma. Especially they are more common in diabetic patients. Pelvic trauma especially those associated with fractures cause urethral injury. Previous trauma increases the risk of infection in urethra. Another common cause is faulty instrumentation into urethra like inadvertent usage of Foleys catheter. Congenital anomalies like posterior urethral valves can cause obstruction urinary stream in children which is mainly studied using micturating cystourethrography that also gives idea over reflux into ureters which is common with posterior urethral valves.

The primary imaging modality for demonstrating the male anterior urethra is fluoroscopic contrast urethrography performed either as a retrograde study via catheter insertion into the distal urethra or as a voiding study to delineate the posterior urethra. The main advantages of the technique are that it has a high sensitivity for the detection of urethral strictures. Interpretation may be hampered by the presence of air bubbles, which may obscure pathology or even provide a false-positive study. In addition, as the balloon of the catheter is inflated in the distal urethra, pathology in this area will not be identified. Urethral ultrasound has not been widely adopted, and is a routine procedure in few centres, yet it addresses several of the shortcomings of the contrast technique [1].

Limitations of retrograde urethrogram, in accurate evaluation of anterior urethral stricture diseases also include variation in the appearance of strictures with position of the patient and the degree of stretch of the penis during the study. It also provides limited information about periurethral structures [2]. Sonourethrogram can be used to overcome the disadvantages of retrograde urethrogram. It lacks radiation which is especially helpful in children.

Advantages of sonourethrogram are relative noninvasiveness, ready availability and lack of ionising radiation exposure, ability of ultrasound to demonstrate the exact length of strictures, ability to define the periurethral tissues, as opposed to contrast urethrography, the presence and degree of periurethral fibrosis shown with a view to guiding surgery.
The main disadvantage with sonourethrogram is lack of information regarding to posterior urethra pathologies. Retrograde urethrogram is better in understanding posterior urethral pathologies. This study helps to understand the advantages of sonourethrogram over retrograde urethrogram, helps to understand the level of utility of sonourethrogram in demonstrating urethral pathologies in comparision with retrograde urethrogram.

\section{Materials and Methods}

Type of study \& period of study: This study was conducted in department of radiology in Kurnool medical college for four years, between 2015 to 2019. This study was basically a comparative study between sonourethrogram and retrograde urethrogram.

Inclusion criteria: Patients with symptoms pertaining to urethral pathologies were selected.

Exclusion criteria: Congenital urethral anomalies and patients with recent trauma were excluded from the study.

Patients with acute urethral infections were also excluded.

Patients with moderate to severe meatal stenosis are excluded.

A total of 60 patients were selected who came for retrograde urethrogram especially those with chronic infection history without any recent trauma or symptoms suggesting acute infection. All patients requested and took informed consent for sonourethrogram.

Study design: Philips, GE and Esoate machines were used to examine male urethra, using probes with frequency $3-12 \mathrm{MHz}$, after filling urethra with saline. Ascending technique was used in sonourethrogram.

Findings were divided into mucosal irregularities suggesting chronic urethritis, strictures either long segmented or short segmented, diverticulae, false passages, periurethral fibrosis, periurethral abscess and urethral calculus. Results were compared with the retrograde urethrogram.

Statistical analysis: Results were analyzed using Cohen kappa statistical measurements rather than simple percent calculations as it takes into account the possibility of agreement occurring by chance. 
Kappa value of less than 0.2 is regarded to as poor agreement.

\section{Results}

Out of 60 patients, 35 patients were of ages between 20-40 years, 20 were between 40-60 years, 05 were either below 20 or above 60 [Table $1]$.

Table 1: Age distribution of patients.

\begin{tabular}{|l|l|}
\hline \multicolumn{1}{|c|}{ Patients age group in years } & \multicolumn{1}{c|}{ Number of patients } \\
\hline Up to 20 & 02 \\
\hline $20-40$ & 35 \\
\hline $40-60$ & 20 \\
\hline Above 60 & 03 \\
\hline
\end{tabular}

Out of 60 , all were having burning micturition, 55 patients complained of obstructing urinary stream, 56 patients had pain in penis. 5 patients were complaining of pus discharge [Table 2].

Table-2: Various symptoms of patients.

\begin{tabular}{|l|l|}
\hline \multicolumn{1}{|c|}{ Symptoms } & \multicolumn{1}{c|}{ Number of patients } \\
\hline Burning micturition & 60 \\
\hline
\end{tabular}

\begin{tabular}{|l|l|}
\hline Obstructing urinary stream & 55 \\
\hline Pain in penis & 56 \\
\hline Pus discharge & 05 \\
\hline
\end{tabular}

Out of 60 patients, 59 had strictures shown in retrograde urethrogram either in penile, bulbar [Fig $1,2,3$ and 4] or membranous parts. Only one patient had membranous urethral stricture in retrograde urethrogram which was not shown in sonourethrogram. All other patients had shown strictures in sonourethrogram. One patient had penile urethral calculus [Fig 5] shown in both sonourethrogram and retrograde urethrogram. Out of 59 who had strictures, 30 were in penile region, 26 were in bulbar region, and in both penile and bulbar parts in 3 patients.

Out of 60 patients, 46 patients had irregular mucosa, 10 patients had false passages, 5 patients had abscess in periurethral region, 15 patients had diverticulae described in retrograde urethrogram [Table 3]. In sonourethrogram, only 08 patients shown false passages and periurethral fibrosis was shown in 25 patients. Periurethral fibrosis was not identified in retrograde urethrogram. Statistical evaluation is shown in Table 3.

Table-3: Comparison of findings between sonourethrogram and retrograde urethrogram and analysis.

\begin{tabular}{|l|l|l|l|}
\hline \multicolumn{1}{|c|}{ Findings } & \multicolumn{1}{|c|}{$\begin{array}{c}\text { Number of patients, shown in } \\
\text { sonourethrogram }\end{array}$} & $\begin{array}{c}\text { Number of patients that can be described in } \\
\text { retrograde urethrogram }\end{array}$ & $\begin{array}{c}\text { Kappa value and agreement } \\
\text { estimate }\end{array}$ \\
\hline Penile urethral strictures & 30 & 30 & 1 -very good \\
\hline $\begin{array}{l}\text { Bulbar urethral strictures } \\
\text { Both penile and bulbar } \\
\text { urethral strictures }\end{array}$ & 26 & 26 & 1 -very good \\
\hline $\begin{array}{l}\text { Membranous urethral } \\
\text { strictures }\end{array}$ & 03 & 03 & 1 -very good \\
\hline Penile urethral calculus & 01 & 01 & $<0.2$-poor \\
\hline Periurethral fibrosis & 25 & 01 & 1 -very good \\
\hline Periurethral abscess & 05 & 00 & - \\
\hline False passage & 08 & 05 & 1 -very good \\
\hline
\end{tabular}
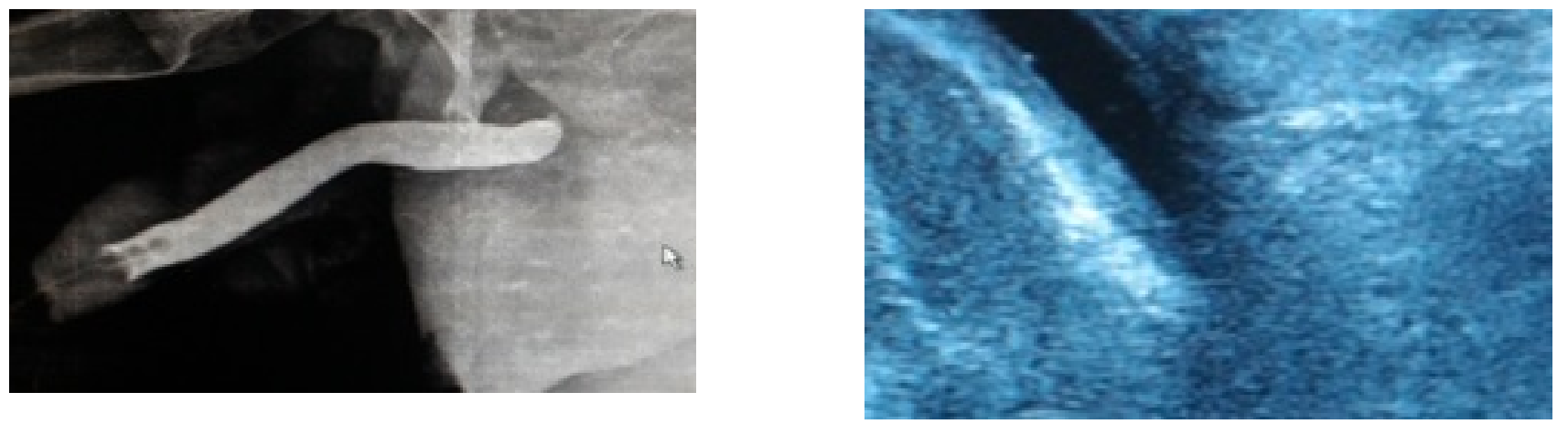
Fig-1. Left side image was an retrograde urethrogram showing stricture in bulbar urethra. Corresponding sonourethrogram image was on right side, showing stricture in bulbar urethral stricture.
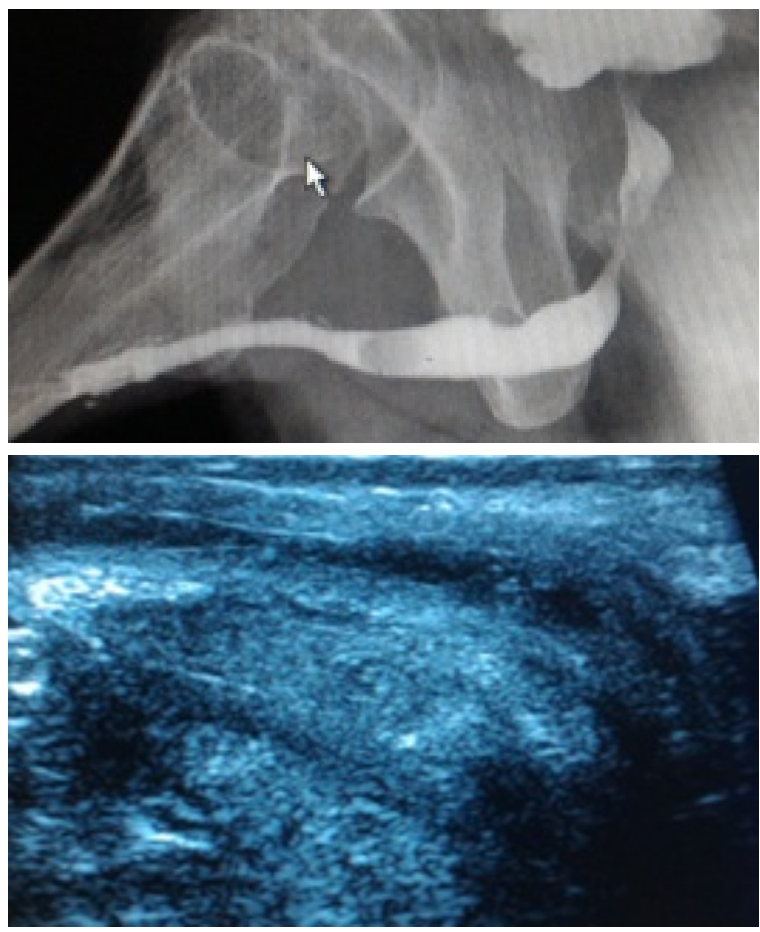

Fig 2. On the left side, Retrograde urehrogram showed long segment stenosis in penile urethra, with multiple diverticuli. Right side image represented corresponding finding in sonourethrogram.

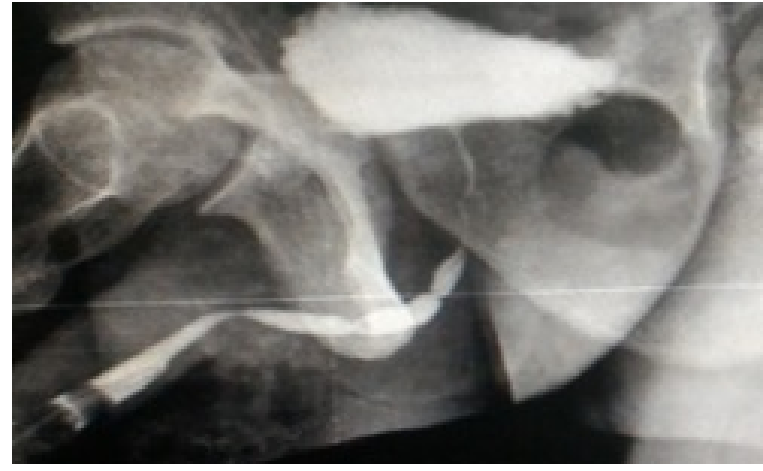

Fig: 3 A

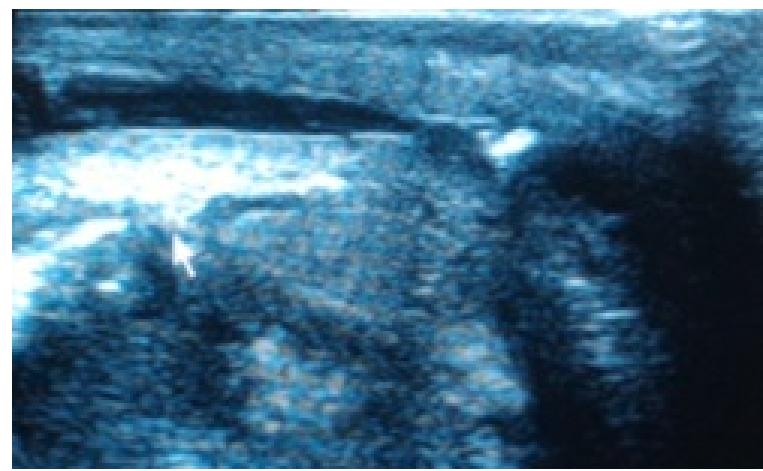

Fig: 3 B

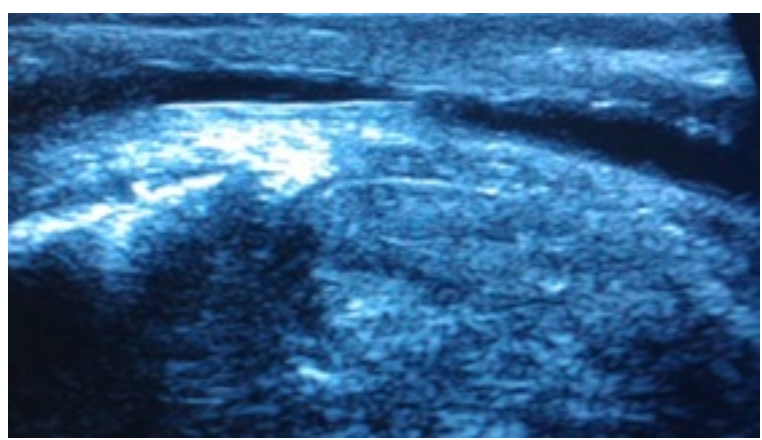

Fig: 3 C

Fig-3. A] retrograde urethrogram showing multiple strictures in penile and bulbar parts. Proximal penile urethra shows a filling defect posterior to stricture representing a calculus.

B] Sonourethrogram shows stricture in proximal penile urethra with periurethral fibrosis and a calculus proximal to stricture.

C] multiple areas of stenosis in penile urethra with periurethral fibrosis.

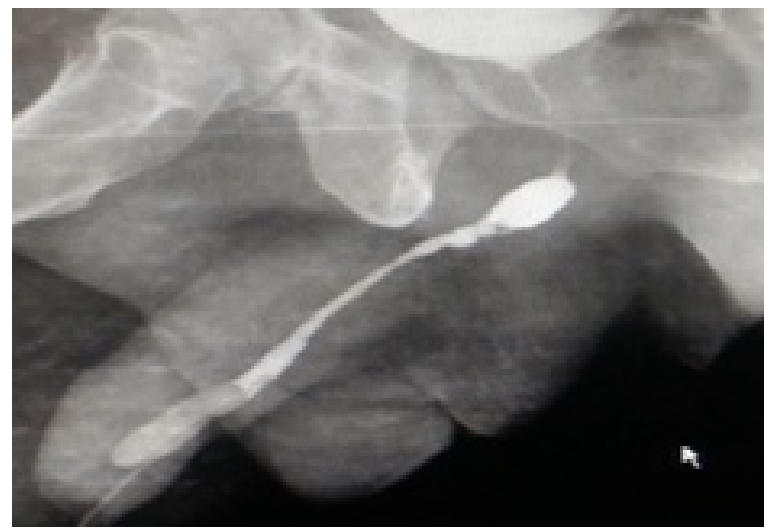

Fig: 4 A

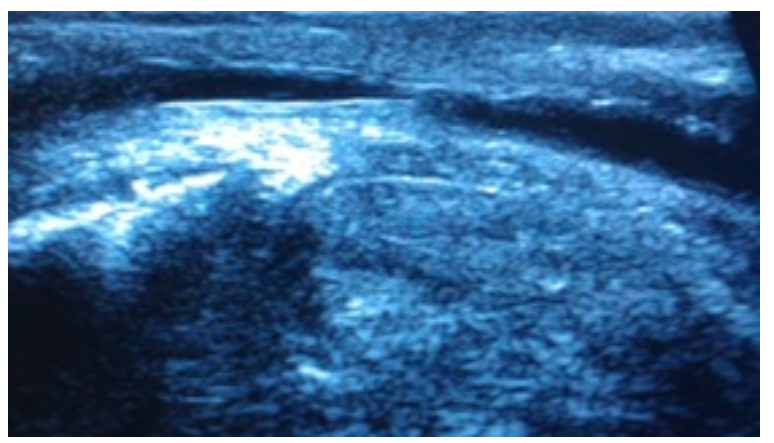

Fig: 4 B 


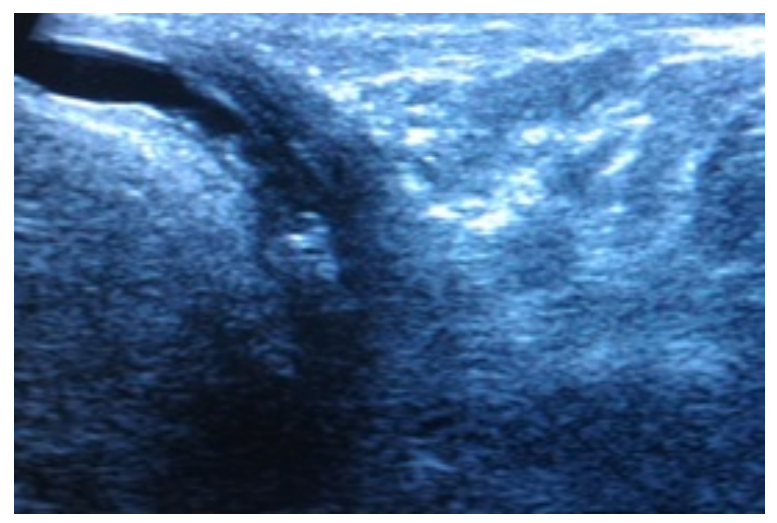

Fig: 4 C

Fig-4 A] Retrograde urethrogram shows long segment stricture in penile urethra, short segment strictures in bulbar urethra. B and C sonourethrogram images showing decreased calibre of penile urethra with strictures in penile and bulbar urethra respectively, with focal fibrosis in bulbar urethra.

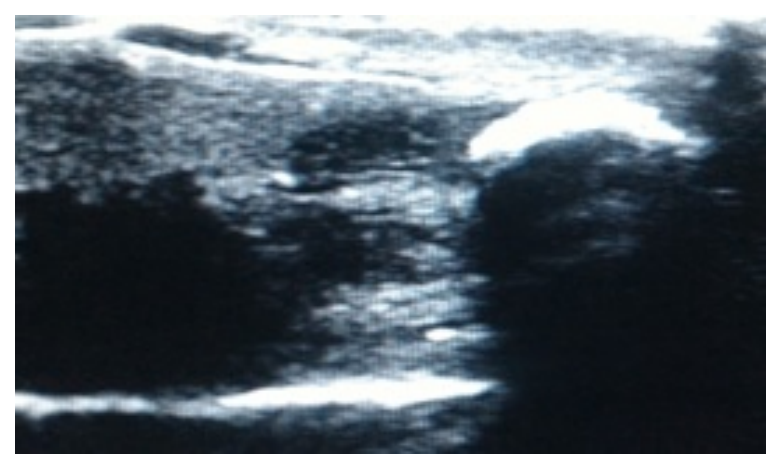

\section{Fig-5: Sonourethrogram showing penile urethral calculus}

\section{Discussion}

Conventionally retrograde urethrogram is used to see urethral abnormalities. It is considered to be gold standard also. It uses radiation, sometimes difficult to perform in meatal stenosis. Interpretation can be hampered by the presence of air bubbles. This cannot give idea regarding to periurethral fibrosis also. To address these drawbacks sonourethrogram can be used as an alternative. In the present study, sonourethrogram had almost $98 \%$ sensitivity regarding to male anterior urethral pathologies compared to retrograde urethrogram.

When compared with conventional RGU, sonourethrography was equally efficacious in detecting anterior urethral stricture diseases.
Further characterization of strictures in terms of length, periurethral pathologies like periurethral fibrosis, diverticula, abscesses, fistulas and false tracts can be performed with relatively greater sensitivity using the sonourethrography [2]. With sonourethrogram, it is difficult to evaluate posterior urethra owing to its inability to scan the urethra in a perpendicular fashion [3]. Ultrasonography can be used as an adjunct to determine the length and degree of spongiofibrosis, and can influence the operative approach [4].

Because sonographic measurements are often longer, new ultrasonic criteria proposed indicate resection and end-to-end anastomosis for adult bulbar stricture measuring up to $25 \mathrm{~mm}$. Ability of sonourethrography to diagnose periurethral pathologies and length of stricture especially in bulbar urethra helps surgeon to plan proper surgical procedure [2]. Periurethral fibrosis is a critical determinant of appropriate therapy and ultimate prognosis [5]. Excessive fibrosis is said to be responsible for high recurrence rates [6].

Ultrasonography of the urethra was shown to directly influence the reconstructive operative approach for anterior urethroplasty in $45 \%$ of cases [1]. Gray scale imaging with doppler seems to be superior to radio urethrography for treatment planning. Sonoure-thrography and MR imaging have been proposed, distending the lumen with simple saline solution instead of iodinated contrast media. They are being used to study the urethral mucosa and the periurethral spongy tissue which can be involved in the urethral pathologies such as strictures, diverticula, trauma, and tumors [7].

Ascending ultrasound technique whereby a catheter was placed distally in a similar manner to contrast urethrography with a balloon inflated in the fossa navicularis and the urethra distended with either saline or, if a conventional urethrogram was also required, contrast medium. Ultrasound was performed using a high-frequency linear array transducer with direct skin contact along the ventral surface of the penis. Subscrotal and perineal views were obtained. There are a number of disadvantages, including the fact that it may not be possible to catheterise the distal urethra, particularly in patients with meatal stenosis or previous surgery. Also, the technique is necessarily invasive, and interpretation may be hampered by the presence of air bubbles, which may obscure pathology or even provide a false-positive study. 
In addition, as the balloon of the catheter is inflated in the distal urethra, pathology in this area will not be identified [8].

Descending approach involved transverse and sagittal views of the urethra using a high-frequency linear array probe. The patient attends with a full bladder and voids into a receptacle. Urethral distension is achieved with the urine stream, which is interrupted by the patient gently clamping the penis between thumb and forefinger during voiding, approximately $2 \mathrm{~cm}$ proximal to the tip after retraction of the foreskin. If needed in selected patients, views of the navicular fossa are obtained while actively voiding.

Advantages of the descending ultrasound technique over the alternative imaging methods are that it is non-invasive, is well-tolerated by the patient, can be performed by a single operator and provides excellent views of the distal urethra, which is especially important in hypospadias assessment [1]. Pitfalls being under distension by an inadequate stream of urine can mimic a long stricture.

A further potential pitfall to be aware of is the fact that care needs to be taken to avoid missing very proximal bulbar strictures. It may be necessary in such cases to perform further voiding views via a perineal approach [1]. Preoperative ultrasonography detect associated abnormalities in children with hypospadias, albeit asymptomatic, irrespective of location of meatus.

No radiation burden to the gonads, particularly in the paediatric population, whether this be stricture disease in the adolescent child or in the context of hypospadias $[8,9,10]$. It is regrettable that the technique is not more widely employed. This may be owing to a general lack of awareness of the facility and utility of the technique among urologists [11]. Also posterior urethral pathologies are difficult to be evaluated with sonourethrogram.

Limitations of the present study was that it cannot be used in patients with complex acute trauma for the fear of aggravating the injury, and in patients with suggesting history of acute urethral infection. It has limited use in evaluating suspected posterior urethral pathologies. And in congenital anomalies like posterior urethral valves, other studies like micturating cystourethrogram is useful. Sonourethrogram has limited role in these conditions.

\section{Conclusion}

Sonourethrogram is easily available, cost effective technique that uses no radiation in the evaluation of male urethra. It is equally effective compared to retrograde urethrogram in the evaluation of male anterior urethral strictures. Periurethral pathologies like fibrosis are better evaluated with sonourethrogram rather than retrograde urethrogram. It is also important in the surgical planning and approach. However, posterior urethral pathologies are difficult to be visualized by sonourethrogram.

This study proves that sonourethrogram is more useful in obtaining information regarding periurethral pathologies like extent of fibrosis accurately than retrograde urethrogram, that helps in surgical planning.

\section{What the study adds to the existing knowledge?}

The present study proves and reinforces that this investigation needed to be performed more often than it is now especially in preoperative stages so that length and extent of fibrosis can be estimated correctly that helps in surgical planning.

Acknowledgment: Author would like to extend his gratitude to Dr. Joji Reddy sir, who is professor and H.O.D of Department of Radiology, and to Dr. Suresh Balla, who is an Associate Professor in the same department for helping out to complete this study.

\section{Reference}

01. Shaida N, Berman LH. Ultrasound of the male anterior urethra. Br J Radiol. 2012;85(1)S94S101.

doi: $10.1259 / \mathrm{bjr} / 62473200$ [Crossref]

02. Hatgaonkar A. A Comparative Study of Sonou rethrography and Retrograde Urethrography in Evaluation of Anterior Male Urethral Strictures. Int J Sci Study. 2014;2(2)5-12.

[Crossref]

03. McAninch JW, Laing FC, Jeffrey RB Jr. Sonourethrography in the evaluation of urethral strictures- a preliminary report. J Urol. $1988 ; 139(2) 294-297$.

doi: $10.1016 / \mathrm{s} 0022-5347(17) 42391-3 \quad$ [Crossref] 
04. Hampson LA, Jack W. McAninch JW, Breyer BN. Male urethral strictures and their management. Nature reviews Urol. 2014;11(1)43-50. doi:10.1038/nrurol.2013.275 [Crossref]

05. Merkle W, Wagner W. Sonography of the distal male urethra--a new diagnostic procedure for urethral strictures- results of a retrospective study. J Urol. 1988;140(6)1409-1411.

doi: $10.1016 / s 0022-5347(17) 42057-x \quad$ [Crossref]

06. Jordan GH, Schlossberg SM, Devine CJ. Surgery of the penis and urethra. In- FC Walsh, $A B$ Retik, ED Vaughan, Jr et al Campbell's urology (7th ed). WB Saunders, Philadelphia.

1998; 3318-3394 [Crossref]

07. Pavlica P, Barozzi L, Menchi I. Imaging of male urethra. Eur Radiol. 2003;13(7)1583-1596.

doi: $10.1007 / \mathrm{s} 00330-002-1758-7 \quad$ [Crossref]

08. Gong EM, Arellano CM, Chow JS, Lee RS. Sonourethrogram to manage adolescent anterior urethral stricture. J Urol. 2010;184(4)1699-1702.

doi: $10.1016 /$ j.juro.2010.03.074 [Crossref]
09. Mitterberger $M$, Christian G, Pinggera GM, Bartsch G, Strasser H, Pallwein L. Gray scale and color Doppler sonography with extended field of view technique for the diagnostic evaluation of anterior urethral strictures. J Urol. 2007;177(3)992-996.

doi: $10.1016 /$ j.juro.2006.10.026 [Crossref]

10. Gupta L, Sharma S, Gupta DK. Is there a need to do routine sonological, urodynamic study and cystourethroscopic evaluation of patients with simple hypospadias?. Pediat Surg Int. 2010;26(10)971-976.

doi: $\quad 10.1007 / s 00383-010-2652-z \quad$ [Crossref]

11. Ferguson GG, Bullock TL, Anderson RE, Blalock RE, Brandes SB. Minimally invasive methods for bulbar urethral strictures- a survey of members of the American Urological Association. Urology. $2011 ; 78(3) 701-706$.

doi: $\quad 10.1016 /$ j.urology.2011.02.051 [Crossref] 